\title{
Frontières
}

\section{Trajectoire de vie et « résilience » des personnes suicidaires}

\section{Monique Séguin}

Volume 12, numéro 1, automne 1999

Suicides, générations et culture

URI : https://id.erudit.org/iderudit/1074502ar

DOI : https://doi.org/10.7202/1074502ar

Aller au sommaire du numéro

Éditeur(s)

Université du Québec à Montréal

ISSN

1180-3479 (imprimé)

1916-0976 (numérique)

Découvrir la revue

Citer cet article

Séguin, M. (1999). Trajectoire de vie et " résilience " des personnes suicidaires. Frontières, 12(1), 11-16. https://doi.org/10.7202/1074502ar

\section{Résumé de l'article}

Comment comprendre l'apport des séparations précoces, des difficultés relationnelles, des problèmes de santé mentale dans la vie des personnes qui se suicident ? Bien que la dépression soit une variable qui explique en partie les comportements suicidaires, il semble y avoir chez les personnes décédées par suicide une trajectoire de vie caractérisée par un cumul de souffrances, de pertes et d'abandons qui se conjuguent à un moment précis de la trajectoire de vie et engendrent chez celles-ci une incapacité d'encaisser encore d'autres pertes. Quelles expériences ont une influence marquante ? De quelle manière ces expériences peuvent-elles avoir une emprise, positive ou négative, au cours de la vie ? L'enjeu consiste à identifier les combinaisons d'événements qui placent les individus à risques de psychopathologie et, en fin de compte, de passage à l'acte suicidaire. On comprend bien que dans cette chaîne d'interactions, les relations d'attachement et l'histoire familiale soient centrales et constituent le début, soit protecteur, soit malheureux du processus d'engrenage.
Ce document est protégé par la loi sur le droit d'auteur. L'utilisation des services d’Érudit (y compris la reproduction) est assujettie à sa politique d'utilisation que vous pouvez consulter en ligne.

https://apropos.erudit.org/fr/usagers/politique-dutilisation/ 


\section{Résumé}

Comment comprendre l'apport des séparations précoces, des difficultés relationnelles, des problèmes de santé mentale dans la vie des personnes qui se suicident? Bien que la dépression soit une variable qui explique en partie les comportements suicidaires, il semble y avoir chez les personnes décédées par suicide une trajectoire de vie caractérisée par un cumul de souffrances, de pertes et d'abandons qui se conjuguent à un moment précis de la trajectoire de vie et engendrent chez celles-ci une incapacité d'encaisser encore d'autres pertes. Quelles expériences ont une influence marquante? De quelle manière ces expériences peuvent-elles avoir une emprise, positive ou négative, au cours de la vie? L'enjeu consiste à identifier les combinaisons d'événements qui placent les individus à risques de psychopathologie et, en fin de compte, de passage à l'acte suicidaire. On comprend bien que dans cette chaîne d'interactions, les relations d'attachement et l'histoire familiale soient centrales et constituent le début, soit protecteur, soit malheureux du processus d'engrenage.

Mots clés: suicide - trajectoire de vie résistance - vulnérabilité - attachement

\begin{abstract}
How can we better understand the contribution of early-life separations, relationship troubles, and mental health problems in the lives of suicidal individuals? Although depression is a variable that partially explains suicidal behavior, it seems that the life path of those who have died as a result of suicide creates an accumulation of suffering, losses and abandons that work together at a specific moment in one's life path and generate within such persons an inability to be resilient to other losses. Which experiences have a major impact? How can these experiences have a hold, either positive or negative, during the course of one's life? The challenge consists of identifying the combinations of events that place individuals at psycopathological risks and, ultimately, lead them to commit suicide. It is well understood that within this chain of interactions, relationships of attachment and family history are crucial and represent the albeit protective and unfortunate beginning of a vicious circle.
\end{abstract}

Key words: suicide - life path - resilience - vulnerability - attachment

\section{Trajectoire de vie et «résilience» des personnes
suicidaires}

Monique Séguin,

professeure au Département de psychoéducation, UQAH, directrice du Laboratoire d'étude sur le suicide et le deuil, Centre de recherche Fernand-Seguin, membre du CRISE.

On sait que les expériences de l'enfance ont un impact sur la vie adulte. Ce constat peut sembler trivial, et sans doute plusieurs expériences très précises, qui ont façonné chacun d'entre nous. pourront être identifiées. Cependant, quelles expériences ont une influence marquante? De quelle manière ces expériences peuvent avoir une emprise, positive ou négative, au cours de la vie? Quels individus seront plus susceptibles d'être marqués par telles ou telles expériences précoces? Nous tenterons ici de mieux cerner comment la qualité d'attachement dans les relations parents-enfants, les expériences précoces de pertes, de séparations, et l'accumulation d'adversité déterminent une trajectoire de vie qui mène ou non à des comportements suicidaires.

Le rôle de la «résilience» dans le développement de la personne aide à mieux comprendre les interactions complexes pouvant mener au suicide. La résilience est la capacité qu'a un individu de réagir d'une façon personnelle devant l'adversité. Pour imager, pensons à la capacité qu'a la personne de rebondir, tel un ressort, lorsqu'elle fait face à une situation d'adversité majeure. Devant une crise ou un événement tragique, les individus ne réagissent pas tous de la même façon et ne peuvent pas rebondir de la même manière, avec la même force ou la même puissance. Certains montrent plus de facilité, alors que d'autres éprouvent davantage de difficulté. La résilience peut donc être perçue comme la capacité de faire face aux situations difficiles, capacité qui varie d'un individu à l'autre. Cela dit, la question demeure entière: qui a la capacité de rebondir et selon quelles conditions?

\section{LE MODÈLE DE VULNÉRABILITÉ ET DE RÉSILIENCE}

La vulnérabilité est définie comme l'ensemble des caractéristiques d'une personne qui fait augmenter les risques de pathologie en présence d'un agent déclencheur ${ }^{1}$. Certaines personnes seront très sensibles à des sources de stress mineures, alors que d'autres seront capables de fonctionner adéquatement, 
même si elles sont exposées à des sources de stress et d'inconfort extrêmes. Le modèle de vulnérabilitérésilience suggère qu'il existe un continuum de vulnérabilité plutôt que deux pôles distincts.

Cette capacité à faire face aux événements difficiles est déterminée par des facteurs biologiques, développementaux et contextuels qui, lors des différentes phases de la vie, interagissent avec les soins, la protection et l'encadrement affectif fournis par le milieu familial, conjugal et social. Certains individus auront dans leur vie moins de chance que d'autres et vivront une série d'expériences difficiles. Certains s'en sortiront parce qu'ils connaîtront des succès importants, ou parce qu'ils auront trouvé sur leur chemin des individus qui sauront leur transmettre un sentiment de confiance, de stabilité et de sécurité. Cependant, d'autres ne vivront pas d'expériences de protection et, au contraire, feront l'expérience de déceptions, d'abandons et de rejets répétés.

Pour comprendre le phénomène de la résilience ${ }^{2}$, il faut adopter une perspective temporelle, tout en tenant compte de la trajectoire de développement de chaque individu. On peut identifier trois composantes de la résilience individuelle qui influenceront soit un renforcement, soit une fragilisation de celle-ci: 1) les relations d'attachement qui s'établissent dans le cours du développement; 2) l'accumulation d'événements dans la vie d'un individu; 3) et l'impact de la maladie mentale.

\section{COMPOSANTE DU DÉVELOPPEMENT}

L'EFFET DES RELATIONS

D'ATTACHEMENT

Depuis les travaux de J. Bowlby ${ }^{3}$ et M. Ainsworth ${ }^{4}$, les liens d'attachement sont considérés comme un aspect central des relations personnelles et $\mathrm{du}$ développement psychosocial. Leur perturbation augmente la vulnérabilité à la dépression ${ }^{5}$. Les chercheurs ont observé que la perte d'un parent pendant l'enfance augmente la vulnérabilité au suicide. La théorie de l'attachement suggère que la qualité de la relation parentale influence la qualité de la relation future avec les pairs. Selon plusieurs auteurs ${ }^{6}$, l'adversité familiale et la psychopathologie parentale sont des variables importantes de risques, et leur présence viendra compromettre l'établissement d'un attachement sécurisant entre parent et enfant. Ainsi, l'incapacité d'établir des liens affectifs stables et sécurisants,

POUR EXPLIQUER L'ÉTABLISSEMENT DE RELATIONS INTERPERSONNELLES

PROBLÉMATIQUES ET CHAOTIQUES.

notamment dans un contexte familial constamment perturbé, place l'individu dans une situation de fragilité, d'anxiété et crée des difficultés dans sa capacité à développer des relations intimes à l'âge adulte. M. T. Greenberg ${ }^{7}$ suggère que les enfants qui ont un attachement anxieux développent un modèle interne de représentation (internal working model) selon lequel les relations d'intimité sont caractérisées par la colère, la méfiance, le chaos et l'insécurité. Pour cette raison, les différentes relations d'attachement au cours du développement ne sont pas indépendantes les unes des autres ${ }^{8}$.

Les premières relations d'attachement vont générer des attentes quant au fonctionnement et à la qualité des relations d'attachement ultérieures. Les expériences de séparation et de rejet placent l'individu dans une situation d'insécurité, ce qui l'amène à se questionner sur sa propre valeur et, ultimement, crée des difficultés dans sa capacité de nouer des liens d'attachement stables et sécurisants. L'incapacité de se sentir intimement lié à d'autres personnes entraîne encore plus d'hésitations et de doutes, sur le sens de la vie. C'est dans cette perspective que les difficultés d'attachement augmentent la vulnérabilité aux épisodes dépressifs.

Plusieurs études confirment l'hypothèse de la transmission de la qualité d'attachement d'un parent à son enfant ${ }^{9}$. D'autres études observent une corrélation entre la qualité des premières relations d'attachement et la manière dont les individus interagissent ultérieurement dans leurs rapports sociaux et avec leur conjoint ${ }^{10}$.

Cependant, les théories de l'attachement laissent entrevoir la possibilité de transformation dans les relations d'attachement lorsqu'un changement se produit ${ }^{11}$. Le mode d'attachement peut se modifier au cours du processus de maturation, à mesure que l'individu devient capable de mieux organiser et d'intégrer les informations complexes qui lui sont disponibles. Ceci provoquera inévitablement des transformations dans sa façon de se représenter à lui-même et de se présenter à l'autre ${ }^{12}$.
La théorie de l'attachement est centrale pour expliquer l'établissement de relations interpersonnelles problématiques et chaotiques.

\section{COMPOSANTE \\ ENVIRONNEMENTALE}

L'ACCUMULATION D'EXPÉRIENCES

D'ADVERSITÉ

On reconnaît que le climat familial est le principal facteur de risque des comportements suicidaires chez les jeunes ${ }^{13}$. Plusieurs types de situations familiales sont identifiés: les relations chaotiques, la violence et les abus physiques, l'alcoolisme des parents, les mésententes conjugales et les comportements suicidaires de l'un des parents ${ }^{14}$. Les abus physiques, l'immaturité et l'incompétence parentale, de même que l'indifférence ou l'absence du père sont des variables qui augmentent le risque de comportements suicidaires des jeunes. À un niveau plus théorique, G. W. Brown ${ }^{15}$ soutient que l'absence d'échanges significatifs d'ordre émotif avec les parents nuit au sentiment d'estime de soi. Ces carences produisent un engourdissement de la vie émotive, trait fréquemment rencontré chez des personnes qui ont des désordres de personnalité et des conduites impulsives.

Les expériences de séparation et de rejet interagissent avec les relations d'attachement tout au long de la vie de l'individu, et elles auront notamment comme conséquence de renforcer ou de fragiliser la capacité d'adaptation dont fera preuve un individu.

Il semble que certaines personnes soient marquées plus sérieusement par leur enfance, tant sur le plan personnel que social. Ainsi, par un ensemble de circonstances, ceux-ci se retrouvent souvent au cœur d'une séquence d'événements qui les laissent de plus en plus vulnérables. Par exemple, si on établit une distinction entre deux types d'événements: ceux sur lesquels les individus peuvent avoir du contrôle et ceux sur lesquels ils ne peuvent en avoir. La situation qui mène une personne à perdre son emploi parce qu'il y a des mises à pied massives dans son milieu de travail n'est pas la même que lorsque la 
personne se fait mettre à pied parce qu'elle est toujours en retard, négligente et distraite, ce qui l'amène à faire des erreurs importantes. Certaines personnes se placent dans des situations à risque et ainsi, il leur arrive plus d'événements qu'à d'autres.

Certaines personnes ont un comportement tel qu'elles multiplient les ruptures au niveau des relations interpersonnelles, professionnelles ou académiques. C'est le cas de la personne vulnérable qui est toujours victime d'événements stressants qu'elle a pour ainsi dire provoqués de façon plus ou moins directe, tels que des peines d'amour à répétition ou des accidents de travail causés par la distraction, etc.

Plus l'individu a vécu de pertes durant son enfance, comme des blessures dues à l'abandon, plus ces expériences d'adversité peuvent créer une impuissance à modifier sa vie et ainsi, les obstacles continuent à s'accumuler sur son chemin ${ }^{16}$.

\section{IMPACT DES MALADIES MENTALES ET HISTOIRE FAMILIALE DE PSYCHOPATHOLOGIE}

Les recherches des dernières années ont démontré la présence de désordres psychiatriques dans les familles où survient un suicide ${ }^{17}$. En ce qui concerne les victimes de suicide elles-mêmes, les études qui ont utilisé l'autopsie psychologique, malgré une méthodologie variable, arrivent à mesurer des taux de dépression. Il semble que l'interaction de facteurs génétiques ou développementaux influence les attitudes et la personnalité, augmentant la probabilité de se placer dans des situations où des événements malheureux de vie se produiront $^{23}$. G.W. Brown et al.24, ainsi que A.T. Harris et al. ${ }^{25}$, mettent en lumière le processus d'engrenage qui engage certaines personnes dans une cascade d'événements les rendant de plus en plus vulnérables à la pathologie. Cet engrenage a souvent comme point de départ une perte ou des situations de rejet grave au cours de l'enfance venant interférer avec l'établissement de relations d'attachement positives. Ces difficultés dans l'établissement de relations d'attachement peuvent se poursuivre tout au long de la vie et créer des insatisfactions ou des échecs relationnels répétés. Ce sont ces échecs répétés qui laissent l'individu toujours un peu plus vulnérable à la dépression et, ultimement, aux comportements suicidaires, lorsqu'il aura à vivre une période d'adversité.

\section{HISTOIRES FAMILIALES \\ ET RÉPÉTITION GÉNÉRATIONNELLE}

La présence de psychopathologie familiale peut entraîner des difficultés dans le développement de relations d'attachement saines et stables. Selon C. Hammen ${ }^{26}$, les femmes qui ont vécu dans leur enfance des expériences négatives avec leurs propres parents seront plus à risque de dépression, pourront

\section{ON RECONNAÎT QUE LE CLIMAT FAMILIAL}

EST LE PRINCIPAL FACTEUR DE RISQUE

DES COMPORTEMENTS SUICIDAIRES CHEZ LES JEUNES

$70 \%$ à $95 \%$ de troubles mentaux chez les suicidés ${ }^{18}$. La comorbidité demeure fort importante à 69 \%, la dépression est le désordre le plus souvent identifié (près de $60 \%$ ), suivi par les abus de substances $(41 \%)$ et les troubles de la personnalité $(41 \%)^{19}$.

Toutefois, la compréhension actuelle de l'étiologie de la dépression n'implique pas qu'une seule vulnérabilité biologique $^{20}$ ou de tempérament, mais aussi une interaction avec des facteurs développementaux ${ }^{21}$ et psychosociaux $^{22}$ qui peuvent refléter une vulnérabilité à la tentatives de suicide dangereuses ont dans leur famille des antécédents de suicide complété. Dans une étude réalisée au Québec, M. Séguin, A. Lesage et M. Kiely $^{28}$ observent que les mères dont le fils est décédé par suicide ont vécu significativement plus de pertes précoces dans l'enfance que les mères de jeunes hommes décédés suite à un accident. De plus, ce sont les mères des jeunes hommes décédés par suicide qui rapportaient le plus de difficultés relationnelles avec leurs fils.

On voit donc ici s'établir le processus d'engrenage observé par l'équipe de G.W. Brown et A.T. Harris ${ }^{29}$ auprès de jeunes mères qui poussent presque inexorablement les jeunes filles qui ont vécu des carences familiales au cours de leur enfance dans un entonnoir menant tout droit à la dépression. La carence parentale entrave l'établissement d'une relation d'attachement sécurisant. C'est souvent le premier maillon de la chaîne qui établit une trame selon laquelle les victimes de carences parentales pendant l'enfance risquent davantage de devenir celles qui ont une faible estime de soi. Une enfance malheureuse pousse souvent une personne à chercher refuge et réconfort dans une relation romantique précoce afin d'oublier les blessures narcissiques profondes. Dans de tels cas, une grossesse précoce peut être un moyen de trouver, auprès de son propre bébé, l'amour dont elle a manqué. Si l'amant ou le futur époux présente un comportement irresponsable ou violent, ceci aura comme conséquence de fragiliser passablement la vie familiale et la possibilité d'élever un enfant dans un milieu sain avec un attachement sécurisant. Ainsi donc, une relation de couple insatisfaisante, la présence d'enfants et le manque d'habiletés parentales peuvent conduire des jeunes mères sur la voie de la dépression et de comportements suicidaires, engageant aussi leurs propres enfants sur la même voie de vulnérabilité reproduisant ainsi le cycle qu'elles ont vécu. On voit donc se tracer les grandes lignes d'une répétition générationnelle d'adversités, à travers l'établissement des relations d'attachement et des relations affectives «insécurisantes».

\section{L'HISTOIRE D'ÉTIENNE}

Dans le cadre des études effectuées au Laboratoire de recherche sur le suicide et le deuil du Centre de recherche Fernand-Seguin, nous nous intéressons à l'histoire de vie des familles dans lesquelles il y a eu perte par suicide. Nous refaisons, avec la participation de 
plusieurs membres d'une même famille, l'histoire de vie, des relations d'attachement et des événements de la vie sur trois générations. En plus, nous recueillons, au moyen d'autopsie psychologique, des informations sur les expériences de séparation précoce dans la vie de la personne maintenant décédée. L'histoire d'une de ces familles permettra d'illustrer nos propos quant à la résilience et l'attachement.

Étienne s'est suicidé au début de 1996, il avait alors 19 ans. Il était le troisième d'une famille de trois enfants: Pierre, l'aîné, a 10 ans de plus qu'Étienne et sa sour, France, a 25 ans, elle est mariée et a un enfant de 7 ans.

Les parents d'Étienne ont eu, dès le début de leur union, une relation pour le moins chaotique. La mère d'Étienne, Claire, s'est mariée à 17 ans, parce qu'elle était enceinte, et elle avait déjà des problèmes d'alcoolisme et de toxicomanie qui se sont constamment aggravés au cours des années suivantes.

Claire a quitté son mari après la naissance de son deuxième enfant. Lorsque France avait cinq ans, Claire a repris la vie commune avec son mari. Un an après, elle donnait naissance à Étienne.

Étienne a passé les quatre premières années de sa vie en famille d'accueil. Il nous a été impossible de comptabiliser le nombre de familles dans lesquelles Étienne a vécu à cette époque. De quatre à six ans, sa vie fut tout aussi chaotique. Tantôt, il vivait avec ses deux

CE SONT CES ÉCHECS RÉPÉTÉS QUI LAISSENT L'INDIVIDU TOUJOURS

UN PEU PLUS VULNÉRABLE À LA DÉPRESSION ET, ULTIMEMENT,

AUX COMPORTEMENTS SUICIDAIRES, LORSQU'IL AURA À VIVRE

UNE PÉRIODE D'ADVERSITÉ.

parents, tantôt en famille d'accueil, tantôt seul avec son père, ensuite un retour en famille d'accueil, de nouveau avec ses deux parents, et ainsi de suite jusqu'à ce qu'il ait l'âge de six ans. C'est à cette époque que sa mère s'est suicidée. À six ans, Étienne fut placé à nouveau en famille d'accueil.

Pendant cette période, son père a développé une nouvelle relation amoureuse avec une femme qui avait, elle aussi, deux enfants.

Vers l'âge de huit ans, Étienne retourna vivre chez son père, la famille reconstituée réunissant alors sa bellemère Jeanne, sa sœur France (qui avait à cette époque 14 ans), son frère (18 ans) qui avait déjà quitté le foyer familial, ainsi que le fils et la fille de Jeanne, qui avaient 15 et 7 ans. De l'âge de huit ans à l'âge de dix-neuf ans, Étienne a vécu avec son père et sa belle-mère. Il a développé peu de liens avec son demi-frère puisque celui-ci a quitté le milieu familial deux ans après la reconstitution de la famille. Sa demi-sour, Caroline, menait une vie de délinquante, sortait avec des délinquants et des revendeurs de drogues. À l'âge de 14 ans, Caroline était placée en centre d'accueil pour jeunes délinquantes.

Dès l'âge de 16 ans, Étienne, qui vivait une relation amoureuse avec Stéphanie, n'était presque jamais chez lui. Il souhaitait fuir le milieu familial, car ses parents passaient leur temps à se disputer au sujet de Caroline. Comme il $y$ avait beaucoup de tension à la maison, il était toujours chez Stéphanie. Ainsi, très tôt, il est devenu très dépendant de Stéphanie; extrêmement jaloux, il soupçonnait tous les garçons à qui Stéphanie adressait la parole, allant même jusqu'à se bagarrer avec l'un d'entre eux.

L'année avant son décès, Étienne a eu une accumulation d'événements malheureux: difficultés scolaires et abandon temporaire de l'école, emploi précaire et mise à pied, ainsi que des difficultés persistantes dans sa relation avec Stéphanie, qui se ponctuait de ruptures et de reprises. Dans les derniers six mois, Étienne pleurait tout le temps, il avait constamment peur d'être abandonné par Stéphanie et la menaçait

\section{Quelques réflexions de... Micheline de Sève \\ professeure au Département de science politique, directrice de I'Institut de recherche et d'études féministes, UQAM.}

Santé, emploi, amis, amour. Deuils multiples. Quand l'espoir s'effiloche, la vie devient friable. Se tuer, ce peut être le choix d'une euthanasie précoce, c'est surtout le constat, rationnel ou non, d'un blocage absolu de l'horizon, humainement insupportable. Quand le quotidien n'est plus que fardeau, le dégoût de soi comme de l'entourage tue. Quelle dignité garder dans ces démarches qui vous ballottent d'un guichet à l'autre, accroc de l'offre illusoire de secours extérieurs, en manque de reconnaissance? Tenu à distance de soi, privé des moyens de l'être, qui viendra repêcher celui ou celle que la détresse affole? Résister à l'appel de mort n'est pas simple dans un milieu en perte de sens, sourd à ce qui sort de l'ordre technique des casiers fonctionnels à gérer. Inconvenant, l'appel à l'aide, le cri dément, s'étrangle dans la ouate du sourire de commande des gens «normaux», ces hommes et ces femmes qui débitent le malheur en tranches, sur rendez-vous, du lundi au vendredi, dans le mépris de l'indifférence ou le ras-le-bol des fonctionnaires de l'âme, piégés par le surmenage. Civilisation dites-vous? 
constamment de se suicider si elle le laissait. La situation devenait chaque jour de plus en plus lourde pour Stéphanie qui ne savait plus comment aider Étienne.

Un événement particulier s'est aussi déroulé au cours de cette année. Étienne a pris la décision de confronter son père au sujet d'un événement dont il avait été victime à l'âge de huit ou neuf ans. À cette époque, il aurait été agressé sexuellement par un voisin et il s'est toujours douté que ses parents étaient au courant de cette situation. Quelques mois avant son décès, il a confronté son père, en sachant que ce dernier savait tout de cet événement; celui-ci lui a répondu que toute cette histoire était du passé et que ça devait en rester là. Finalement, trois jours avant son décès, Stéphanie le quitta pour quelqu'un d'autre.

Maintenant, regardons l'histoire de France, la sœur d'Étienne.

Elle aussi a vécu les difficultés liées à un milieu familial chaotique et au suicide de sa mère. Elle aussi a été placée dans une famille d'accueil, dont elle dit ne pas avoir de souvenir avant l'âge de 11 ans. Vers cet âge, elle fut agressée sexuellement alors qu'elle vivait dans une famille d'accueil. À l'âge de 14 ans, elle retourna vivre avec son père et sa belle-mère. De 14 à 16 ans, elle a habité avec son père et sa belle-mère, de qui elle dit: "C'est juste la blonde de mon père».

À l'âge de 16 ans, elle devient enceinte et se marie. Elle a maintenant 24 ans, elle a un fils de 7 ans et est toujours mariée avec le même homme. Elle n'a jamais fait de dépression et n'a jamais eu de comportement suicidaire.

Comment expliquer que ces vies qui peuvent sembler assez parallèles, du moins pour les premières années (suicide de leur mère, même encadrement familial, même vie chaotique, mêmes placements en série en famille d'accueil et agression sexuelle), aient un aboutissement si différent?

On peut penser que France soit plus résiliente que l'était Étienne. France a sans doute eu des éléments de protection que n'a pas eus Étienne: le tempérament peut-être, le fait qu'elle soit une fille, sans doute; l'attachement au père était plus positif et même s'il était idéalisé, il a quand même pu servir de protection, et son partenaire a sans doute joué aussi un rôle de protection, compte tenu de sa stabilité et de son rôle d'encadrement auprès de France. De plus, le fils de France fut possiblement un élément fort positif dans la vie de sa mère. Alors
qu'Étienne avait un attachement au moins à Stéphanie, qui était caractérisé par la méfiance, l'insécurité et l'enchevêtrement dans un rapport de fusion-idéalisé. Il n'a sans doute pas été capable de faire face à l'abandon, ce qui a peut-être accru le sentiment de faible estime de soi, la colère, le rejet, et a déclenché le passage à l'acte.

\section{ET LA PROTECTION}

Malgré tout, certaines personnes sont extrêmement résilientes; tout comme les chats, elles retombent toujours sur leurs pattes! Le modèle de vulnérabilité-résilience suppose qu'il y a interrelation entre les événements difficiles et les facteurs de risque, d'une part, et les attributs personnels et les facteurs de protection, d'autre part. C'est dans la compréhension des facteurs de protection que les intervenants peuvent agir pour renforcer la résilience de personnes potentiellement vulnérables.

Il existe trois grandes catégories de facteurs de protection. La première est constituée des «dispositions personnelles de l'individu», comme le tempérament, les habiletés intellectuelles, l'utilisation de l'humour, le lieu de contrôle, la qualité des stratégies de «coping» et la connaissance de soi $^{30}$. La deuxième catégorie comprend «la cohésion et la chaleur familiale», qui fait référence à des qualités qui favorisent un milieu familial sain et positif dans lequel nous retrouverons des adultes ayant des compétences parentales, un climat qui favorise l'établissement de relations d'attachement positives, et la présence d'une relation de confidence entre les conjoints $^{31}$. Enfin, la troisième catégorie renvoie à «la disponibilité et à l'utilisation du soutien social» qui inclut une relation significative avec un adulte et la présence d'expériences positives comme des succès scolaires ${ }^{32}$. Ces éléments de protection contribuent à développer un ensemble de cognitions positives à l'égard de soi, rehaussent le sentiment d'estime de soi et diminuent la détresse émotionnelle. Des liens positifs d'attachement peuvent s'établir à différentes phases du développement et servir de support à la résilience, face à une situation grave d'adversité.

\section{DISCUSSION}

On voit bien que l'histoire d'Étienne met en perspective la manière dont les interactions sont complexes et comment les différents événements de la vie se conjuguent à des moments précis et font en sorte qu'une personne est capable ou non de rebondir lors d'une perte impor- tante. Certaines personnes sont plus résiliantes et plus capables de rebondir que d'autres. Certaines aussi ont à faire face, malheureusement, à un plus grand nombre de situations difficiles alors que d'autres ont des vies assez protégées ou calmes, et leur résilience est moins mise à l'épreuve.

Si une proportion importante de personnes décédées par suicide ont vécu des expériences difficiles au cours de l'enfance, ont manifesté un type d'attachement dysfonctionnel et ont été victimes d'expériences de vie majeures avant leur décès, il reste que la majorité des personnes qui ont connu ou vivent ces expériences difficiles ne se suicident pas. L'enjeu consiste à identifier la combinaison d'événements, tels que des expériences nocives durant l'enfance, des modèles d'attachements malsains et des événements évoquant des expériences particulièrement traumatiques qui placent les individus à risque de psychopathologies et, ultimement, de passage à l'acte suicidaire. On comprend bien que, dans cette chaîne d'interactions, les relations d'attachement et l'histoire familiale sont centrales et constituent le début, soit protecteur, soit malheureux, du processus d'engrenage. 
Notes

1 M. RUTTER, «Resilience in the Face of Adversity», British Journal of Psychiatry, 1985 , no 147 , p. $598-611$

2 M. RUTTER, «La résilience: quelques considérations théoriques», dans BOLOGNINI et al., Préadolescence: Théorie, recherche et clinique, Paris, ESF Éditeur, 1994, p. 147-157

3 J. BOWLBY, «Process of Mourning», The International Journal of Psycho-Analysis, 1961, no 42, p. 317-340.

J. BOWLBY, Attachment and Loss. Volume I: Attachment, London, Hogart, 1973.

J. BOWLBY, Attachment and Loss, Volume II: Separation, New-York, Basic, 1980.

4 M. AINSWORTH, Patterns of Attachment, Hillsdale, NJ, Erlbaum, 1978.

5 E.H. ERIKSON, Childhood and Society, New York, Norton, 1950.

6 K. LYONS-RUTH, D. Zoll, D. CONNELL et H.V. GRUNEBAUM, «Family Deviance and Family Disruption in Childhood», Development and Psychopathology,1989, no 1, p. 219-236.

S.J. SPIEKERS et C.L. BOOTH, «Maternal Antecedents of Attachment Quality», dans J. Belsky, Clinical Implications of Attachement, Hillsdale, NJ, Erlbaum, 1988.

7 M.T. GREENBERG, M.L. SPELTZ et M. DEKLYEN, «The Role of Attachment in the Early Development of Disruptive Behavior Problem", Development and Psychopathology, 1993, no 5, p. 191-213.

8 J. BOWLBY, Attachment and Loss, Volume II: Separation, ibid.

9 I. BRETHERTHON et al., "Attachment: the Parental Perspective», Infant Mental Health Journal, 1989, no 10, p. 203-221.

D. COHN, «Child-Mother Attachment of Six-Year Olds and Social Competence at School», Child Development, 1989, no 61, p. 152-162.

C. GEORGE et J. SOLOMON, «Internal Working Models of Caregiving and Security of Attachment at Age Six», Infant Mental Health Journal, 1989, no 10, p. 222-237.

K. GROSSMAN et al., "Maternal Attachment Representations as Related to Patterns of Infant-Mother Attachment and Maternal Care During the First Year», dans R.A. HINDE et STEVENSON-HINDE, Relationships within Families, Oxford, Clarendon Press, 1988.

M. MAIN, N. KAPLAN et J. CASSIDAY, "Security in Infancy, Childhood, and Adulthood: A Move to the Level of Representation", Monographs of the Society for Research in Child Development, 1985 , no 50, p. 66-104.

10 G.C. ARMSDEN et M.T. GREENBERG, «The Inventory of Parental and Peer Attachment: Individual Differences and their Relationship to Psychological WellBeing in Adolescence», Journal of Youth and Adolescence, 1987, no 16, p. 427-453. P.M. CRITTENDEN, «Family Patterns of Relationship in Normative and Dysfunctional Families", Development and Psychopathology, 1994, no 3, p. 491-512.
11 M.H. VANIJZENDOORN, "Of the Way we Are: On Temperament, Attachment, and the Transmission Gap», Psychological Bulletin, 1995, vol. 117, no 3, p. 404410.

12 W.R. BEARDSLEE, «The Role of SelfUnderstanding in Resilient Individuals: The Development of a Perspective», American Journal of Orthopsychiatry, 1989, vol. 59, no 2, p. 266-278.

13 D.A. BRENT et al., "Suicidal Behavior Runs in Families: A Controlled Family Study of Adolescent Suicide Victims", Archives of General Psychiatry, 1996, no 53, p. $1145-1152$.

D.M. ADAMS, J.C. OVERHOSLER et A. SPIRITO, «Stressful Life Events Associated with Adolescent Suicide Attemps, Canadian Journal of Psychiatry, 1994, vol. 39, no 1, p. 43-48.

14 N. FARBEROW, "Youth Suicide: A Summary", dans M.L. PECK, N. FARBEROW et R.E. LITMAN, Youth Suicide, New York, Springer, 1985.

15 G.W. BROWN et A.T. HARRIS, Life Events and Illness, New York, Guilford, 1989.

16 T. HARRIS, G.W. BROWN et A. BIFULCO, "Loss of Parent in Childhood and Adult Psychiatry Disorder: the Role of Lack of Adequate Parental Care», Psychological Medicine, 1986, no 16, p. 641-659.

17 D. BRENT, "Facteurs de risque associés au suicide à l'adolescence», Prisme, 1995, vol. 5 , no 4, p. 360-371.

$18 \mathrm{~J}$. BESKOW, B. RUNESON et U. ASGARD, «Psychological Autopsies: Methods and Ethics», Suicide and LifeThreathening Behavior, 1990, vol. 20, p. 307-323.

19 A.D. LESAGE et al., "Suicide and Mental Disorders: A Case-Control Study of Young Adult Males», The American Journal of Psychiatry, 1994, no 151, p. 1063-1068.

20 P. MCGUFFIN, R. KATZ et J. RUTHERFORD, "Nature, Nurture and Depression: A Twin Study», Psychological Medicine, 1991, no 21, p. 329-335.

21 M. RUTTER, «Temperament, Personality and Personality Disorder», British Journal of Psychiatry, 1987, no 150, p. 443-458.

22 G.W. BROWN et al., "Social Support, Self-Esteem and Depression», Psychological Medicine, 1986, no 16, p. 813-831.

23 P.E. BEBBINGTON et al., «The Camberwell Collaborative Depression Study I. Depressed Probands: Adversity and the Form of Depression", British Journal of Psychiatry, 1988, no 152, p. $754-765$

24 G.W. BROWN, I.Z. ADLER, et A. BIFULCO, "Life Events, Difficulties and Recovery from Chronic Depression», British Journal of Psychiatry, 1988, no 152, p. 487-498.

25 T. HARRIS, G.W. BROWN et A. BIFULCO, «Loss of Parent in Childhood and Adult Psychiatry Disorder: the Role of Lack of Adequate Parental Care», Psychological Medicine, ibid.
26 C. HAMMEN, D. BURGE et K STANSBURY, «Relationship of Mother and Child Variables to Child Outcomes in a High-Risk Sample: A Causal Modelling Analysis», Developmental Psychology, 1990, no 26, p. 24-30.

27 D. BRENT, "Facteurs de risque associés au suicide à l'adolescence», Prisme, ibid.

28 M. SÉGUIN, A. LESAGE et M. KIELY, «History of Early Loss Among a Suicide Group», Crisis, vol. 16, no 3, p. 121-125.

29 G.W. BROWN, I.Z. ADLER, et A. BIFULCO, «Life Events, Difficulties and Recovery from Chronic Depression», British Journal of Psychiatry, ibid.

30 W.R. BEARDSLEE, «The Role of SelfUnderstanding in Resilient Individuals: The Development of a Perspective», American Journal of Orthopsychiatry, ibid.

31 P.M. CRITTENDEN, «Family Patterns of Relationship in Normative and Dysfunctional Families", Development and Psychopathology, 1995, no 3, p. 491-512. M. HEIKKINEN, H. ARO et J. LÖNNQVIST, «Recent Life Events, Social Support and Suicide», Acta Psychiatry Scandinavia, 1994, no 377, p. $65-72$.

32 S.S. LUTHAR et E. ZIGLER, «Vulnerability and Competence: A Review of Research on Resilience in Childhood», American Journal of Orthopsychiatry, 1991 , vol. 61, no 19, p. 6-22. 